\title{
Horizons/Théâtre
}

Revue d'études théâtrales

12 | 2018

Les dramaturgies arabes et l'Occident

\section{Tayeb Saddiki (1939-2016), un pont entre les dramaturgies arabes et l'Occident. Témoignage d'un entretien}

Angela Daiana Langone

\section{OpenEdition}

Édition électronique

URL : http://journals.openedition.org/ht/454

DOI : $10.4000 /$ ht. 454

ISSN : 2678-5420

Éditeur

Presses universitaires de Bordeaux

\section{Édition imprimée}

Date de publication : 1 janvier 2018

Pagination : 170-183

ISSN : 2261-4591

\section{Référence électronique}

Angela Daiana Langone, «Tayeb Saddiki (1939-2016), un pont entre les dramaturgies arabes et l'Occident. Témoignage d'un entretien », Horizons/Théâtre [En ligne], 12 | 2018, mis en ligne le 01 janvier 2019, consulté le 19 juillet 2019. URL : http://journals.openedition.org/ht/454 ; DOI : 10.4000/ ht. 454

\section{(c) (i) $\odot$}

La revue Horizons/Théâtre est mise à disposition selon les termes de la Licence Creative Commons Attribution - Pas d'Utilisation Commerciale - Pas de Modification 4.0 International. 


\section{Angela Daiana Langone}

Angela Daiana Langone est chercheuse à I'Université de Cagliari (Italie), où elle enseigne la langue et la littérature arabes, ainsi que chercheuse associée à l'IREMAM de l'Université d'Aix Marseille. Elle a obtenu le Prix d'Encouragement à la Recherche 2016 décerné par l'Académie des Sciences d'Outre-mer de Paris et a publié plusieurs ouvrages concernant la littérature populaire arabe et la production écrite en arabe dialectal. Parmi ses publications, on rappellera en particulier son livre Molière et le théâtre arabe. Réception moliéresque et identités nationales arabes (Berlin, De Gruyter 2016).

Mail : langone@unica.it

Résumé: Tayeb Saddiki (1939-2016) est universellement reconnu comme le monstre sacré du théâtre marocain. Ses activités de dramaturges ont consisté en plusieurs adaptations des œuvres théâtrales de nombreuses traditions différentes, ainsi que dans la création de pièces en arabe (dialectal marocain

Abstract: Tayeb Saddiki (1939-2016) is universally recognized as the giant of Moroccan theater. His theatrical activities consisted in many adaptations of dramas belonging to different traditions, as well as the creation of many productions in both (Moroccan and Standard) Arabic and French. The following et arabe littéraire) et français. Les pages qui suivent transcrivent un entretien accordé en juin 2004 où il parle du lien entre ses expériences personnelles et sa vie artistique.

Mots-clés: Tayeb Saddiki, Théâtre, Maroc

pages transcribe an interview given in June 2004 where he speaks about the relationships between his personal experiences and his artistic life.

Keywords: Tayeb Saddiki, Theater, Morocco 


\section{Tayeb Saddiki (1939-2016), \\ un pont entre les dramaturgies \\ arabes et l'Occident. \\ Témoignage d'un entretien}

\section{Introduction}

LORSQU'ON PARLE DE DRAMATURGES qui ont su conjuguer magistralement les techniques occidentales et le patrimoine arabe traditionnel, on ne peut pas négliger les ouvrages d'un monstre sacré du théâtre au Maroc: Tayeb Saddiki (al-Ṭayyib al-Ṣiddīqī), né à Essaouira le 5 janvier 1939 et disparu à Casablanca le 5 février 2016.

Il a été adaptateur de nombreuses pièces étrangères, comme celles du théâtre de l'absurde de Beckett et de Ionesco, ou du théâtre italien de Goldoni et de la Commedia dell'Arte, ou encore du théâtre de Molière et du théâtre russe. Mais il a également été engagé dans le vaste travail de récupération de la tradition pré- et para-théâtrale arabe et notamment marocaine ${ }^{1}$.

Cette contribution présente une interview que Tayeb Saddiki m’a accordée dans son habitation à Casablanca en juin 2004, jamais publiée auparavant et que j'avais gardée dans un tiroir pendant plus de dix ans. La récente perte humaine de ce géant du théâtre mondial, ainsi que le sujet de ce numéro de la revue consacré aux relations entre les arts de l'Orient et de l'Occident, mais aussi les échanges scientifiques avec son directeur, le collègue Omar Fertat qui a eu la chance de connaître en profondeur Saddiki, m'ont donné l'envie de reprendre ce matériel pour la publication, dans l'espoir de contribuer à la connaissance de ce pionnier du théâtre arabe, véritable passeur entre les différentes cultures.

Cette interview retrace les phases du parcours artistique et personnel de Saddiki : de son enfance à Essaouira, ville modèle de tolérance et de coexistence entre les religions, à la découverte de Marrakech et sa merveilleuse place Jemaa el-Fna, un véritable théâtre de plein air; son passage fortuit de l'architecture au théâtre; son réseau de relations avec les autres intellectuels et quelques-unes de ses idées politiques sur le monde arabe et sur la Méditerranée jusqu’à arriver à sa fascination pour la calligraphie. 


\section{L'entretien}

\section{Votre père était un faqīh, pourquoi avez-vous choisi le théâtre ?}

Tout ce que j'ai fait dans ma vie, je l'ai fait par accident. Mais il y a une chose qui m’a frappé : à cinq ans, mon père m'a amené à Marrakech et j'ai découvert Jemaa el-Fna. Je l'ai découverte presque à la tombée de la nuit, j'étais très fasciné mais je ne comprenais pas bien encore. Je n'ai pas choisi le théâtre, je crois que c'est le théâtre qui m'a choisi. Moi, je voulais faire de l'architecture. J'étais l'un des rares bilingues - il y avait deux écoles, l'une avec des cours en français, l'autre avec des cours en arabe - et je traduisais pour les uns et les autres. Un jour, un comédien est tombé malade, j’ai joué à sa place ; un jour on n'avait plus de metteur en scène, j'ai fait la mise en scène... Puis c'est tout. Je n'ai jamais eu de stratégie préconçue pour faire du théâtre. Je l'ai fait un peu en tâtonnant. Mon premier maître a été surtout Jean Vilar au Théâtre National Populaire, à l'époque où il y avait Gérard Philipe, Philippe Noiret, Jeanne Moreau... On le sait après que c'étaient des grands, cela ne se sait jamais sur le moment.

\section{Votre ville d'origine est Essaouira. Quels sont les souvenirs liés à} votre ville?

Lorsque les gens visitent Essaouira, ils disent tout simplement: « est-ce que tu as remarqué que la ville est très propre? », « est-ce que tu as remarqué aussi le rapport avec les étrangers? ». Tu n'es jamais étranger à Essaouira. Personne n'est étranger à Essaouira. Je ne dis pas ça parce que c'est ma ville, mais parce que c'est la vérité. Et les gens sont tolérants, sont ouverts.

Lorsque j'ai quitté Essaouira, j'avais sept ans. Donc, bien entendu, j'ai des souvenirs. D'ailleurs, je le dis dans mon roman Mogador, fabor ${ }^{2}$. J'avais des souvenirs assez étonnants parce qu'à cinq ans déjà je parlais l'arabe et l'hébreu mais je ne faisais pas la différence. La moitié de la population était juive et l'autre moitié musulmane. Les gens cohabitaient, les gens mangeaient ensemble, les gens discutaient, ils riaient ensemble, ils pleuraient ensemble. La plupart des juifs marocains sont plutôt des Berbères judaïsés. La religion juive existait au Maroc bien avant l'arrivée des Arabes, mais c'est le problème palestinien qui a fait que les communautés se sont un petit peu séparées. Quand je vais en Israël, je rencontre des tas de juifs qui me connaissent. Il y a plus de 600000 juifs marocains qui vivent à la marocaine. Il y a des salons à la marocaine avec la photo de Mohammed V et de Hassan II. Ils mangent à la marocaine, le jour de shabbat, ils s'habillent en djellaba à la marocaine, en 
tarbouche rouge et ils jouent de la musique marocaine et entre eux quand ils se parlent, ils se parlent en arabe marocain. Ils ne parlent pas entre eux en hébreu. Du moins, jusqu’à cette génération. L'autre qui arrive ou va arriver va estomper tout cela.

Alors ce sont des souvenirs enrichissants aussi bien que douloureux. J'étais très étonné quand je suis arrivé à Casablanca, d'abord de trouver que la ville était très sale par rapport à Essaouira. Essaouira est une des villes les plus propres du monde. Les méchantes langues disent que c'est parce qu'il y a plus de vent. Bon, c'est le premier souvenir et je ne vous cache pas que quand je rêve d'elle, la plupart du temps je vois une rue où il $y$ a trois juifs qui prisent du tabac, le tabac qu'on met dans le nez, avec des mouchoirs blancs et des taches rouges. Ça c'est la première image d'Essaouira qui me vient à l'esprit.

\section{Pourquoi avez-vous choisi l'iqtibās, l'adaptation pour le public marocain de quelques pièces étrangères ?}

D’abord quand je suis arrivé au théâtre, il y avait très peu d'auteurs de théâtre au Maroc et même dans le monde arabe. Donc, adapter pour moi c'est me confronter au théâtre international pour apprendre à écrire le théâtre. Ce sont Molière, Carlo Goldoni, Eduardo Scarpetta, Lope de Vega qui m'ont appris à écrire le théâtre. Mais cela a été très vite, environ une dizaine d'années. Après j'ai compris que je ne pouvais jamais jouer des pièces françaises mieux que la Comédie française, des pièces italiennes mieux qu'à la Scala de Milan, mieux que Giorgio Strehler et ainsi de suite. Donc je me suis dit: Il faut que je trouve quelque chose d'autre. Comment faire pour trouver un théâtre original ? Je me suis rapporté à tout ce qui se dit de populaire, les proverbes, les contes, et tout ça, et j’ai rencontré Jemaa el-Fna. Et naturellement j'ai compris à travers Jemaa el-Fna qu'il fallait aller dans ce sens-là. Puis, je me suis dit: «Bon, c'est bien de travailler sur le théâtre populaire mais il faut des textes forts » et je me suis souvenu que mon père qui était un grand lettré - je n'ai aucun mérite, je suis né dans les livres - nous lisait les maqāmāt, ce qu'on appelle 《les séances », et j'ai commencé à adapter cela, j'ai commencé à adapter des textes qui nous unissent, parce qu'il n'y a pas d'unité arabe, mais nous sommes unis par la langue, uniquement par la langue. Nous sommes très loin les uns des autres.

4. Parmi les auteurs que vous avez adaptés, vous avez choisi des russes, par exemple Gogol...

Oui, j'ai adapté Le Journal d'un fou de Gogol ${ }^{4}$. L'histoire du Journal d'un fou est très simple. Je suis très-très pro Gogol. Je souhaite un jour monter Les âmes 
mortes, même si c'est une œuvre qui n'est pas achevée. Je pense que Gogol et Tchékhov sont deux auteurs russes qui sont très proches de notre culture. Par exemple, Tchékhov, quand il écrit, il décrit un monde qui est un peu rural et qui devient citadin. Cela m'a énormément intéressé. Quand il parle de toutes les fautes d'orthographe des enseignes dans la rue, cela me rappelle le Maroc.

Gogol, lui, c'est sur la corruption. Une chose que nous vivons encore. La première fois que j’ai monté an-Neqša c'était par amitié pour un comédien algérien qui s'appelle Sid Ahmed Agoumi ${ }^{5}$. Il avait quitté le Théâtre National Algérien. Il était un petit peu abattu et il est venu chez moi pendant deux ans. Et je lui ai monté cette pièce-là qui ne nous coûtait pas cher. C'était en 1970. Le sujet était très intéressant - l'aliénation - et moi, j'avais vécu avec une psychiatre, Rita El Khayat ${ }^{6}$ - elle écrit beaucoup - elle m'a beaucoup encouragé à parler de cette sorte de maladie. L'aliénation dans notre société est une chose qui m’a toujours fasciné parce que notre société ne rejette pas les aliénés. Il y a un seul asile dans tout le Maroc. Le fou est protégé dans sa famille, il est protégé dans sa cité, dans sa tribu, dans son village. Il n'est pas rejeté, au contraire, celui qui a perdu la raison est presque un saint. Parce qu'en général, il est inoffensif.

Ce qui m'a intéressé, c'est l'histoire d'un petit fonctionnaire, un tout petit fonctionnaire qui s'occupe des porte-plumes du Directeur; assez extraordinaire comme fonction. Rien que pour cela, il devient fou. Une situation tout à fait dramatique que vit cet homme : il y a là vraiment une rupture avec la réalité et il se crée sa propre réalité : la fille du Directeur, le chien de la fille, il discute avec les chiens ... c'est extraordinaire parce que c'est un monde reconstitué qui n'a aucun rapport avec la réalité, mais ce nouveau monde ne manque pas d'intérêt. Infișām, la schizophrénie, c'est le détachement. Après cela, j'ai repris la pièce en 1998 et en 2001 avec Mohamed Zouhir, un metteur en scène qui a joué le rôle d'Ahmed Agoumi. La dernière représentation s'est déroulée à Grenoble en 2001, pendant l'année du Maroc. Nous avons joué au Maroc et en France avec Mohamed Zouhir, avec Agoumi, c'est tout le Maghreb. J'ai écrit an-Neqša dans un dialecte compris par tous les Maghrébins.

\section{Et puis, vous avez consacré une place considérable à Molière...}

Molière a été pendant très longtemps absolument terrorisé par les gens de l'Église. L'Église, qu'elle soit chrétienne, musulmane ou juive, c'est pareil. En l'honneur de Molière, j'ai écrit une pièce que j'ai appelée Molière ou «Pour l'Amour de l'Humanité $\gg^{7}$. Parce qu'à un certain moment, Dom Juan se promenait avec Sganarelle dans une forêt et arrive un mendiant qui demande l'aumône. Et Dom Juan lui demande: « Mais qu'est-ce que tu fais pendant 
toute la journée ? ». Il lui dit: «Je prie Dieu ». Et Dom Juan lui répond: «Alors, va chez Dieu pour qu'il te donne à manger ». Et il sort un louis d'or et il lui dit: « Je te donne ça, si tu jures ». Et Dom Juan lui jette la pièce et lui dit: « Je te donne ça pour l'amour de l'humanité ». C'est beau.

Moi, je ne considère pas Molière comme un écrivain uniquement français. Il est méditerranéen. Je ne considère pas les auteurs italiens comme étant des Européens, ils sont méditerranéens. Et ceux qui ont donné le plus, on ne le voit pas uniquement dans le théâtre : regardez le cinéma italien, si ce n'est pas méditerranéen!

\section{D’autres dramaturges que vous avez aimés ?}

J'ai beaucoup d'amis parmi les morts. À côté de Molière, il y a Shakespeare. Et puis Ionesco. Lors du dernier Ramadan, j'ai traduit trois pièces de Ionesco. Et Beckett. Aucune troupe au monde n'a jamais autant joué En attendant Godot ${ }^{8}$ que moi, près de quatre cents fois. Aucune, même pas en Europe.

Parmi les dramaturges arabes, il est difficile de citer des noms. Je dirais Sa 'd Allāh Wannūs, ainsi que Ezzedine Madani. Il n'y a pas beaucoup de dramaturges arabes et de dramaturges en général. Shakespeare est mort il y a trois siècles, l'Angleterre attend toujours un autre Shakespeare et je crois qu' ils l'attendront encore très longtemps.

\section{Quel est, à votre avis, le rapport entre l'Orient et l'Occident ?}

Les Arabes sont les porteurs de la civilisation grecque. La Grèce est le pays qui a donné la civilisation occidentale, mais c'est le pays le plus oriental : il n'y a qu'à entendre les chants, il n'y a qu'à connaître ma sœur, c'est plus qu'une sœur, Irène Papas, et il n'y a qu'à lire Eschyle. C'est l'Occident maintenant qui va nous donner des leçons, moi, je veux bien en recevoir. Il y a eu le tremblement de terre à $\mathrm{Al} \mathrm{Hoceïma}{ }^{9}$, les médias français disaient : «Ah, les secours sont arrivés en retard ». Pendant ce temps-là, en France, il y a eu 15000 vieillards qui sont morts à cause de la canicule. En France, ils nous donnent des leçons, alors qu'ils ont de l'eau. 15000 . Qu'ils ne nous donnent pas de leçons. À mon avis, le grand problème de l'Occident c'est qu'il nous considère toujours inférieurs. Nous n'avons pas les moyens qu'a l'Occident. Mais ce n'est pas pour cette raison qu'il faut nous considérer toujours en bas de l'échelle.

Il y a le monde arabe. Moi, je me sens plus proche d'un Portugais, d'un Espagnol que d'un arabe du Moyen Orient. Pourquoi? Ce qui unit le monde arabe, c'est la langue arabe, bien entendu le classique, mais rien de plus, pas même sur un plan de vie, de rapport ou de tolérance. Al-Maǵrib al-Aqșà, c'est le far west, l'occident le plus lointain. Par exemple, les Irakiens ce sont des 
Mésopotamiens, les Égyptiens, ce sont des farā 'ina (Pharaons), les Syriens, ce sont des Levantins, ainsi que les Libanais. D’ailleurs pour moi, le Liban n'existe pas, c'est la Grande Syrie, ce qu'on appelle le Croissant Fertile qui englobe la Syrie, le Liban et la Jordanie. Le Liban et la Jordanie ce sont des états-majors de l'occupant, ils ont dessiné ça. Ce n'est pas grave ça. Ce qui est grave, c'est de croire qu'il y a une unité arabe politiquement parlant. L'unité arabe n'existe pas et ça n'existera jamais. Chacun a sa culture qui est très différente. Le Maghreb a, grosso modo, la même culture. Le Machrek a grosso modo la même culture. Seuls les imbéciles parlent de nation arabe, il n'y a pas de nation arabe, il n'y aura jamais une nation arabe et Dieu merci. Parce que le monde arabe est un monde tellement déchiré. Mais quand même, le monde arabe, c'est Averroès, c'est Ibn Sina, c'est l'invention du zéro, c'est la philosophie. Au Moyen Âge, il y avait une culture arabe commune. Pourquoi ? Parce qu'ils avaient assez de possibilités pour réfléchir à un monde nouveau. Les Occidentaux, qu' ils soient Italiens ou Français, n'ont jamais lu les Grecs sinon à travers les traductions faites par les Arabes. Bon, chaque civilisation, comme chaque être humain, connaît le sommet et la décadence. Nous avons tout connu, dans le monde arabe.

En Italie, je me suis toujours senti beaucoup plus chez moi qu'en France parce que dans la rue, les gens sont comme les Arabes. Ils ont cette espèce d'ouverture, cet humour. Parce que les gens ne savent pas que le monde arabe a beaucoup d'humour et de sens artistique. Si un jour j'allais à Rome, j'irais sur la place Bernini ${ }^{10}$, je fumerais une cigarette à onze heures et je prendrais un Martini, en hommage à Fellini. Un jour, on lui a demandé de faire un film publicitaire sur le Martini. Il était dans un train, avec une bouteille de Martini devant lui, il passait par Pise et la bouteille s'est penchée. Ça c'est fabuleux. C'est le génie Fellini, «La Strada », ça ... c'est fabuleux. Et si un jour, les autorités marocaines m'empêchaient de vivre au Maroc, je vivrais en Italie! Alors que la France, la France c'est mon sang, tout ce que tu veux... mais l'Italie c'est mon cœur.

\section{La question de la langue dans le théâtre arabe}

Il ne faut pas oublier que la littérature arabe qu'elle soit populaire ou écrite, fascine les gens. Le mot est presque sacré chez les Arabes. On considère le verbe comme la chose la plus importante. Jorge Luis Borgès dit dans l'Aleph, qu'un groupe d'Arabes racontait que lorsqu' ils étaient en Chine, ils avaient vu quelque chose d'étonnant, ils avaient vu des gens dans un endroit qui disaient des choses bizarres, ils parlaient de palais alors qu'il n'y avait pas de palais, ils parlaient d'épées alors qu'ils n'avaient qu'un bâton. Ils disaient des choses 
bizarres. Quelqu'un a demandé: «Est-ce qu'ils étaient plusieurs ? » La réponse : « Oui ». «Et alors, ce sont des fous. Pourquoi dire cela alors qu'un seul peut tout dire? » C'était le théâtre chinois qu' ils décrivaient. Ils n'avaient pas compris ce que c'était du théâtre ${ }^{11}$. C'est pour cela que le conteur est la base de tout théâtre arabe. Cela demeure toujours. Et la langue est presque sacrée.

La langue arabe n'est pas une langue de mots, c'est une langue de racines. Je te dis par exemple al-fā', wa-l-ğìm wa-r-rā'. Furğa, c'est « spectacle », Fağr « aube », infiğār « explosion » et farğ «le sexe féminin ». Dans le sexe féminin il y a l'aube, il y a l'explosion ... il y a surtout al-farăg, la joie... c'est une langue de racines, c'est l'exotérique, al-bātin.

Vous avez le 'alif qui est le signe divin, vous avez le $b \bar{a}$ ' qui est la soumission, avec un tout petit point, après il y a le $t \bar{a}$ ' une soumission avec deux petits points, puis le $t \bar{a}{ }^{\prime}$ avec trois petits points mais il est déjà loin de Dieu. Après il y a un groupe que j'appelle « un groupe de vierges folles et repliées », c'est le ğim, le $h \bar{a}^{\prime}$ et le $h \bar{a}^{\prime}$, tout ça descend, tout, tout l'alphabet sauf le lām. Le lām : il s'insurge presque contre Dieu, contre le 'alif. Mais quand on dit lām-'alif, c'est l'impossibilité de s'insurger contre la divinité. Il vous donne: lā, c'est l'impossibilité, c'est comme ça que ça fonctionne.

L'hébreu, c'est la langue la plus facile à apprendre quand on connaît l'arabe.

Le dialecte est le parler du peuple. Je le respecte et c'est un enrichissement. Moi, je considère que le dialecte est une partie intégrante de la langue arabe. Le dialecte c'est le dire populaire. Moi, je ne crois pas en l'unicité, même pas politiquement. Dans les institutions, heureusement, ils disent que tout le monde est égal devant la justice. Parce que les salopards, ils attendent les pauvres au tournant. Les puissants peuvent être illégaux, personne ne les touchera, donc il faut quand même faire la part des choses.

Le dialecte, c'est la langue du terroir ainsi que l'amazigh. C'est un enrichissement linguistique. Et puis l'ouverture : au Maroc, on parle le français, l'espagnol et l'anglais. Il ne faut pas oublier que nous sommes à $13 \mathrm{~km}$ de l'Europe. Quand vous êtes à Tanger, vous voyez les lumières de Tarifa ou d'Algésiras. Pour nous c'est essentiel. Maintenant quelqu'un qui ne parle que trois langues, il est presque illettré.

Moi, ce que je n'aime pas du tout c'est de parler en mélangeant les langues. Quelqu'un qui te dit: « gāài nmši la-restaurant», «ntlāqaw à quatorze heures », etc. Moi, je suis horripilé. Ou on parle en arabe ou on parle en français. 
Dans le domaine théâtral, surtout les comédies légères sont toujours en arabe dialectal. En classique, c'est toujours des gens qui veulent intellectualiser la pensée, naturellement ils préfèrent écrire en arabe classique. Heureusement que depuis une dizaine d'années, nous avons commencé à imprimer les pièces, à les éditer. Et ça, c'est très important, parce que le livre est un support de mémoire. Depuis environ dix ans, la télévision repasse d’anciennes pièces qui ont été oubliées, qui ont été jetées. C'est aussi un travail de mémoire. Et un théâtre qui n'a pas de mémoire est un théâtre mort.

Je n'ai écrit que six pièces en français. Ce n'est pas beaucoup. Par exemple, Un incident technique indépendant de notre volonté: j'ai joué cette pièce-là ${ }^{12}$ qui a été soutenue par l'Ambassade de France, c'est une pièce écrite en français. Et l'ambassadeur était un grand ami à moi. Il n’a pas pu venir - j'ai donné quarante ou cinquante représentations à Casablanca, il n'a pas pu venir. Même à Rabat, il n’a pas pu être présent parce qu'il était en déplacement à Paris, mais un jour il nous a rejoint à Tétouan, à $15 \mathrm{~km}$ de l'Espagne. Et c'était extraordinaire, parce qu'il y avait beaucoup de monde, mais on avait un problème, un problème technique, un problème de lumière. Puis j'ai dit: «Bon, pour ne pas faire attendre les gens, je vais improviser quelque chose. $\gg$ Et je suis monté sur scène en disant: « Mesdames et messieurs, la formule typique c'est "un incident technique indépendant de notre volonté" qui s'applique malheureusement à nous ce soir, à cause de l'électricité, nous allons donc commencer avec un peu de retard. Ne nous en veuillez pas. » Et par la suite, l'électricité a fonctionné à nouveau dans le théâtre. Alors je me suis dit: «Voilà, c'est ma nouvelle pièce. $\gg$ Et je l'ai écrite en une semaine.

Je préfère m'approcher beaucoup plus de l'arabe classique parce que cela me permet de jouer de Casablanca jusqu'aux portes du Pakistan. Si je joue en arabe marocain, à part au Maghreb, où est-ce que vous voulez que je joue?

\section{9- À propos de cela, vous avez osé l'expérience d'un théâtre interarabe...}

Oui, effectivement... Avec Mme al-Achqar ${ }^{13}$, on dirigeait tous les deux une troupe : j'avais des Irakiens, des Palestiniens, des Syriens, des Libanais, des Algériens et des Marocains. Cette troupe représentait un peu le microcosme du monde arabe. Je me suis aperçu très vite qu'on avait les mêmes problèmes que le monde arabe. Les Syriens ne pouvaient pas jouer en Iraq, les Irakiens en Syrie... J'ai fini malgré tout par les faire jouer, mais avec beaucoup de difficultés. Et je me souviens d'une rencontre avec Saddam Hussein, quand il y avait la guerre entre l'Iran et l'Irak. Quand j'ai rencontré Saddam Hussein, parce qu'on a donné cinquante représentations à Bagdad, il m'a reçu et je lui ai demandé : « Monsieur le Président, j'ai beaucoup de problèmes avec les deux 
Tayeb Saddiki (1939-2016), un pont entre les dramaturgies arabes et l'Occident...

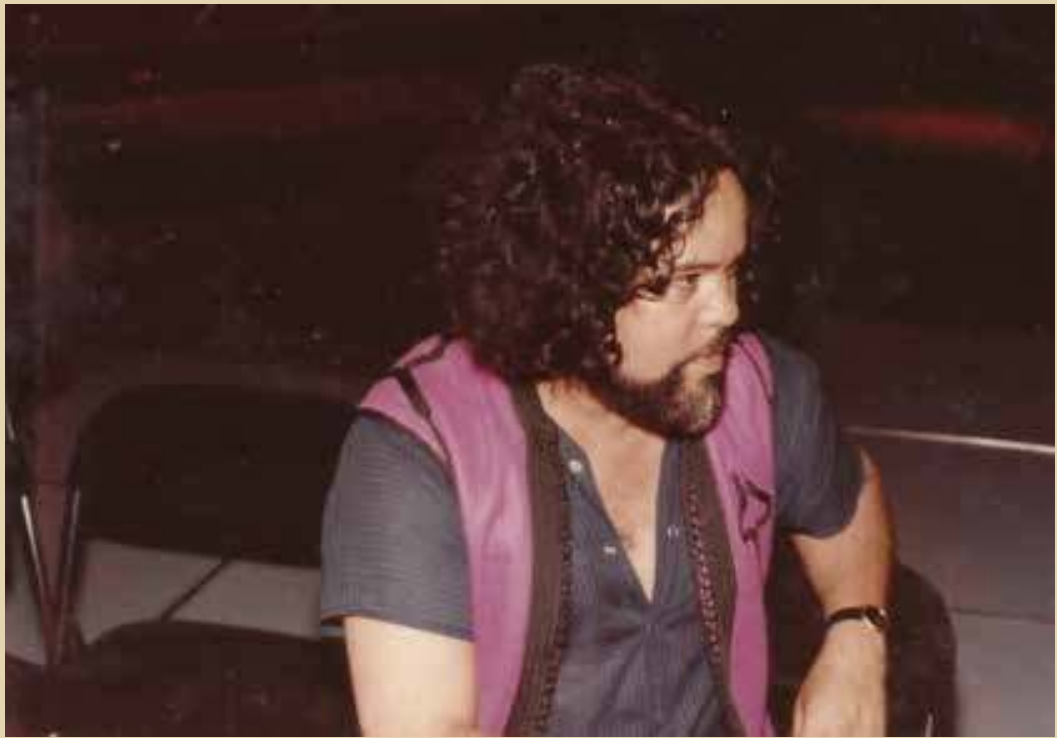

Tayeb Saddiki (1939-2016)

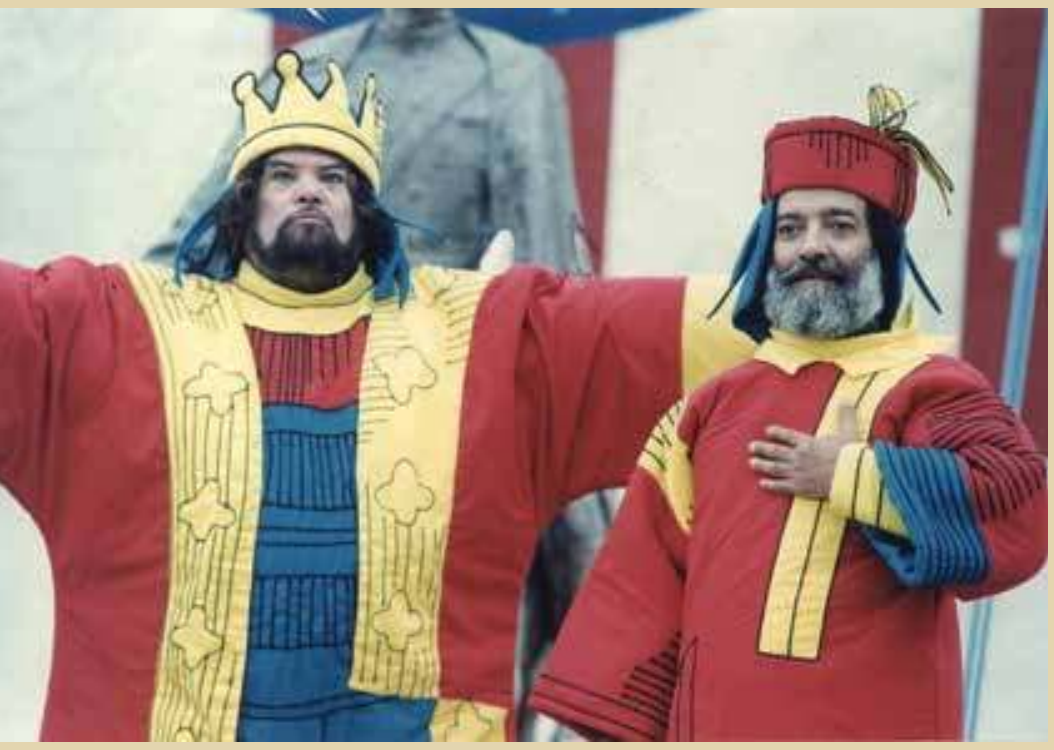

Tayeb Saddiki dans Wa Laou Kanat Foula (même une fève) 
Les dramaturgies arabes et l'Occident

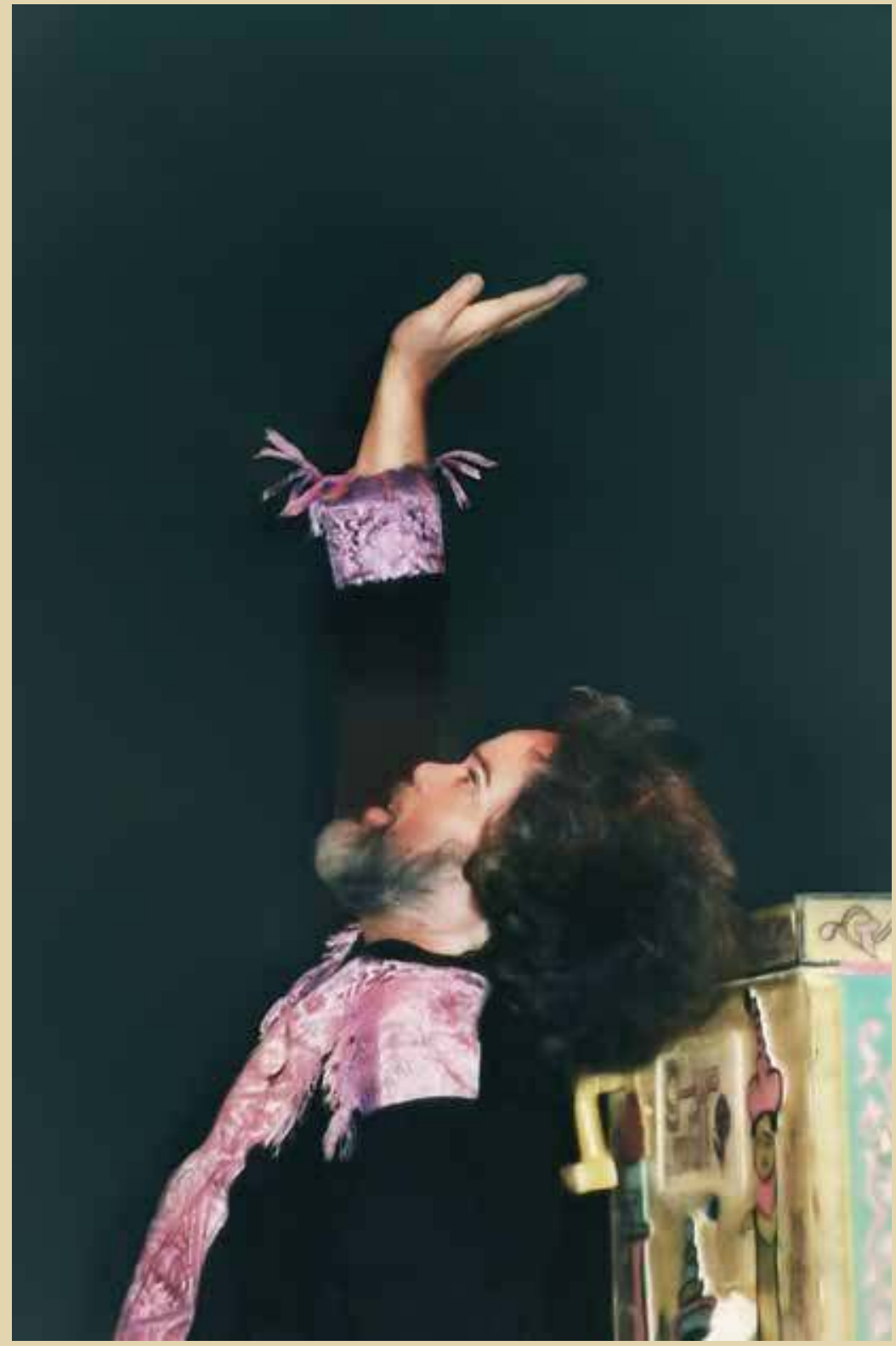

Tayeb Saddiki dans Les Sept grains de beauté 
comédiens irakiens parce qu'à chaque fois qu'ils doivent sortir du pays, ils doivent demander l'autorisation. » Et je lui ai donc demandé de leur donner une autorisation de six mois, un an. Il m'a dit: «C'est interdit chez nous. » Je lui ai dit : «Comment ça ? ». Il m'a dit: « Il est interdit d'exporter les cerveaux irakiens à l'extérieur du pays. » Je lui ai répondu: «Mais monsieur le Président, la plus grande preuve que ces gens-là n'ont aucun cerveau, c'est parce qu'ils ont choisi le théâtre! Moi, qui vous parle, est-ce que vous croyez que j'ai un cerveau, moi? » Et il est parti. Et son fils m'a dit qu'il riait aux éclats.

Cette troupe-là était le reflet de la situation du monde arabe et des rapports des Arabes entre eux. Malheureusement, on n'a pas pu continuer cette expérience d'abord parce qu'elle coûtait très cher. Figurez-vous que pour aller à Londres, nous étions six Marocains, donc Casa-Londres, puis une Algérienne, Alger-Londres, sans parler des Libanais ... Rien que pour les billets d'avion... On avait demandé un financement à quelques émirs. On avait fini par trouver un financement arabe, mais il a duré de huit à neuf mois, on a dépensé énormément d'argent. L'expérience a duré un an, un an et demi. J'écrivais avec Nidal al-Achqar le scénario, la musique était composée par Mounir Béchir ${ }^{14}$, l'irakien. Un Libanais créait les costumes. Cela a été la première et la dernière expérience dans le monde arabe, c'était en 1986-1987. On l'avait appelée Alf hikāya wa-hikāya fī sūq 'Ukā $z^{15}$.

\section{0 - Quel est le rapport des jeunes marocains avec le théâtre ?}

Je crois que les gens sont passionnés par le théâtre. Peut-être que maintenant il y a un petit vide, comme dans tous les pays du monde avec les satellites, ça n'encourage pas beaucoup les gens à aller au théâtre. Mais moi, je dis « ce n'est pas très grave que les gens ne viennent pas au théâtre ». Il faut que nous allions, nous, vers les gens.

Il faut revoir tout au théâtre : les heures du spectacle, les salles, la durée du spectacle. Maintenant, il n'est plus possible de jouer trois heures une pièce de théâtre! Moi, j'appelle les gens à jouer des pièces qui durent une heure pour qu'on les joue deux fois par jour. Il n'est plus possible de jouer pendant trois heures. Vous savez qu'est-ce qu'on dit? Nous sommes maintenant - regardez le langage - en zap, en zip, en clip, en clap, en rip, en fit... Plus personne ne dit un « contrat synallagmatique », c'est du chinois, ça devient du chinois. Donc, il faut revoir les lieux du spectacle et les heures du spectacle.

Il faut aller dans les écoles, il faut aller jouer dans la rue, pourquoi pas? Il faut créer des événements. Mais être dans un théâtre et attendre que le public 
vienne c'est impossible. Et c'est partout pareil, cette histoire-là, ce n'est pas uniquement au Maroc.

\section{L'avenir du théâtre au Maroc}

Donnez-moi une boule de cristal et je vous dirai l'avenir du théâtre au Maroc. Moi, je n'en sais rien mais je suis très optimiste. D'abord, nous, en tant que peuple du sud on souhaite, on n'a que des souhaits. Moi, je suis un optimiste endurci. Dans la vie, je ne vois pas forcément le mal. Moi, je suis très optimiste pour le Maroc. C'est l'un des rares pays qui est vraiment ouvert sur tout. C'est un pays de tolérance, il reste encore du chemin à faire, mais je crois que nous sommes sur la bonne voie parce que le Maroc a des atouts. D'abord sa population, et puis surtout l'ouverture d'esprit des gens. Vous savez, manger en plein jour durant le Ramadan c'est une chose intolérable mais quand il y a quelqu'un qui est fou durant le Ramadan, il est protégé par la population. Le fou n'est pas rejeté comme je vous l'ai dit. Et je crois que cela est très important.

\section{2 - Quel est votre rapport avec l'art dans la vie quotidienne?}

Moi, je suis un producteur. Je confectionne un produit à vendre que ce soit une toile - je suis calligraphe - ou que ce soit le théâtre. L'histoire de l'art pour l'art, ça c'est de la m... Moi, je suis un professionnel. On fabrique un produit à vendre. Pourquoi ? Parce que l'artiste doit vivre $c$ Roûte que $c$ Roûte ${ }^{16}$.

Tout ce que je fais est de la dérision. Je ne me prends pas toujours au sérieux. Je n'ai aucun message à délivrer à l'humanité. Je ne peux pas monter une pièce que je n'aime pas. Je monte ce que j'aime et que je veux partager avec les autres, tout simplement. C'est dans la pratique théâtrale que je suis le plus heureux. Pour une raison très simple : c'est l'art du faux. Vous prenez une canne et vous dites « ceci est mon épée », vous mettez la canne entre vos jambes et vous dites « je pars en voyage » et tout le monde sait que c'est un cheval. Personne dans la salle ne vous dit: «Ce n'est pas vrai. » Prenez une tragédie comme Macbeth, et vous faites pleurer les gens avec tous ces éléments qui sont faux: le théâtre est l'art le plus surréaliste qui existe. À la fin de la pièce, le traitre ou l'ennemi est puni, le rideau tombe, les gens applaudissent, le rideau se lève, le mort se lève et salue les gens. Ce n'est pas formidable ça ? C'est fantastique, il n'y a aucun art qui peut faire ça. Un mort qui salue les gens, c'est quand même extraordinaire! 
Tayeb Saddiki (1939-2016), un pont entre les dramaturgies arabes et l'Occident...

\section{Notes}

1. Pour une biographie détaillée de Tayeb Saddiki, voir en particulier, entre autres, JeanFrançois Clément, Tayeb Seddiki, l'homme de théâtre, Éditions Les Infréquentables, Marrakech, 2012.

2. Tayeb Saddiki, Mogador, fabor, Éditions Eddif, Casablanca, 2004.

3. Parmi ses pièces tirées des maqāmāt, voir aṭ-Ṭayyib aṣ-Ṣiddīqī, Maqāmāt Badī az-Zamān al-Hamadānī [Les maqāmāt de Badī‘ az-Zamān al-Hamadānī], al-Būkīlī, al-Qunayța, 1998.

4. Sous le titre d’an-Naqša («Le déclic »). La pièce, écrite en darija, se trouve dans le recueil aț-Ṭayyib aṣ-Șiddīīi, talātatu nuṣuṣ masraḥiyya [Trois textes théâtraux], Manšūrāt Wizārat at-Taqāfa, ar-Ribāt, 2003, qui réunit en outre le texte d'al-Mufattiš, adaptation d'un autre ouvrage de Gogol, Le Revizor, ainsi que Vülpün, adaptation du Volpone de Ben Jonson.

5. De son vrai nom Sid Ahmed Méziane (Bologhine, 1940-).

6. Rita El Khayat (Rabat, 1944-) est une auteure très prolifique. Parmi ses essais: Rita E1 Khayat, Une Psychiatrie Moderne pour le Maghreb, L'Harmattan, Paris, 1994; Rita E1 Khayat, Métissages Culturels, Éditions Aïni Bennaï, Casablanca, 2003. Parmi ses romans les plus connus: Rita El Khayat, La Liaison, Éditions Aïni Bennaï, Casablanca, 2006.

7. Tayeb Saddiki, Molière ou «Pourl'Amour de l'Humanité», Éditions Eddif,Mohammedia, 1994.

8. Pièce adaptée par Saddiki avec le titre de F̄̄ intizāar Mabrūk ["En attendant Mabrouk»]. La pièce n'a toujours pas été imprimée et le texte n'est que manuscrit, comme le témoigne Omar Fertat, «Tayeb Saddiki, tel que je l'ai connu », L'opinion, 12 février 2016.

9. La nuit du 24 février 2004. Le séisme de magnitude 6,3 sur l'échelle de Richter a causé plus de 550 morts et plus de 900 blessés. Pour des données plus précises, voir, entre autres: OCDE, Études de l'OCDE sur la gestion des risques au Maroc, Paris, Éditions OCDE, p. 165.

10. Saddiki se référait probablement à la Place Navone où se trouve la Fontaine des Quatre-Fleuves bâtie par Gian Lorenzo Bernini au Xviı ${ }^{\mathrm{e}}$ siècle.

11. Jorge Luis Borgès, "La quête d'Averroës », in L'Aleph, trad. Roger Caillois et René L.-F. Durand, Paris, Gallimard, p. 69-77.

12. Tayeb Saddiki, Un incident technique indépendant de notre volonté, Eddif, Casablanca, 2001.

13. Nị̣āl al-Ašqar (Dīk al-Mahdī, 1934-) a joué un rôle prééminent, notamment à partir des années 60, pour le renouvellement du mouvement théâtral libanais et arabe en général.

14. Munīr Bašīr (Mossoul 1930 - Budapest 1997) a été l'un des plus grands joueurs de oud de tous les temps. Pour des approfondissements, voir Chérif Khaznadar, « Munir Bashir, l'homme ", Cahiers d'ethnomusicologie 11, 1998 in http://journals.openeditions. org/ethnomusicologie/1637 [date de dernière consultation: 23/04/2017].

15. «Les Mille et Une Nuits au marché de 'Ukāẓ ».

16. Jeux de mots avec le verbe « coûter » et le substantif « croûte». 